PROCEEDINGS OF THE

AMERICAN MATHEMATICAL SOCIETY

Volume 130, Number 5, Pages 1355-1364

S 0002-9939(01)06240-2

Article electronically published on December 27, 2001

\title{
APPROXIMATION WITH INTERPOLATORY CONSTRAINTS
}

\author{
H. N. MHASKAR, F. J. NARCOWICH, N. SIVAKUMAR, AND J. D. WARD \\ (Communicated by David R. Larson) \\ Dedicated to Professor Ambikeshwarji Sharma, on the occasion of his 80th birthday
}

\begin{abstract}
Given a triangular array of points on $[-1,1]$ satisfying certain minimal separation conditions, a classical theorem of Szabados asserts the existence of polynomial operators that provide interpolation at these points as well as a near-optimal degree of approximation for arbitrary continuous functions on the interval. This paper provides a simple, functional-analytic proof of this fact. This abstract technique also leads to similar results in general situations where an analogue of the classical Jackson-type theorem holds. In particular, it allows one to obtain simultaneous interpolation and a nearoptimal degree of approximation by neural networks on a cube, radial-basis functions on a torus, and Gaussian networks on Euclidean space. These ideas are illustrated by a discussion of simultaneous approximation and interpolation by polynomials and also by zonal-function networks on the unit sphere in Euclidean space.
\end{abstract}

\section{INTRODUCTION}

Let $\Xi=\left\{x_{j, n}: 1 \leq j \leq n, n=1,2, \ldots\right\}$ be a triangular array of distinct points in $[-1,1]$. Given a bounded function $f$, let $L_{n}(f, \Xi, x)$ denote the unique polynomial of degree at most $n-1$ satisfying the interpolatory conditions $L_{n}\left(f, \Xi, x_{j, n}\right)=f\left(x_{j, n}\right), j=1, \ldots, n$. A well-known theorem of Faber (see, for example, [2, p. 215]) asserts the existence of a continuous function $f$ on $[-1,1]$ for which $\limsup _{n \rightarrow \infty}\left\{\max _{-1 \leq x \leq 1}\left|f(x)-L_{n}(f, \Xi, x)\right|\right\}=\infty$. So obtaining a convergent interpolatory process demands further refinements, which can be achieved by pursuing any of the following tacks: (i) placing further restrictions on $f$ (e.g. [12, p. 10]), (ii) interpolating at special sets of nodes (e.g. [1, 5]), or (iii) relaxing the requirement that the interpolant be of minimal degree $n-1$. This last issue has been investigated by several mathematicians, including Erdős, Szabados, and Vertesi; see, for instance, [3, 15, 17, 16. In particular, Szabados has proved the following [15: Suppose $n$ is a positive integer, and let $x_{j, n}=\cos \theta_{j, n}$, where $0 \leq \theta_{1, n}<\ldots<\theta_{n, n} \leq \pi$ and $\min _{1 \leq j \leq n-1}\left(\theta_{j+1, n}-\theta_{j, n}\right)=: \eta_{n}$. Given any continuous function $f:[-1,1] \rightarrow \mathbb{R}$, there exists a polynomial $p_{n}(f, x)$ of degree $2 m \leq c / \eta_{n}$

Received by the editors July 12, 2000.

2000 Mathematics Subject Classification. Primary 41A17, 42C10, 41A05; Secondary 65D32, $65 \mathrm{D} 05$.

The research of the first, third, and fourth authors was supported by grants DMS-9971846, DMS-9706583, and DMS-9971276, respectively, from the National Science Foundation. The work of the second and fourth authors was supported by grant F49620-98-1-0204 from AFOSR. 
(where $c>0$ is an absolute constant) such that $p_{n}\left(f, x_{j, n}\right)=f\left(x_{j, n}\right), 1 \leq j \leq n$, and $\max _{-1 \leq x \leq 1}\left|f(x)-p_{n}(f, x)\right| \leq K \inf \left\{\max _{-1 \leq x \leq 1}|f(x)-R(x)|\right\}$, where the infimum is taken over the space of all polynomials $R$ of degree at most $2 m$, and $K$ is a constant which is independent of $f$ and $n$. Similar results regarding polynomial interpolation may be found in [16. The proofs of these results are usually quite complicated, and depend upon the particular situation at hand. One motivation of the current paper is to provide a simple, yet general, method to derive such results.

We also wish to extend this theory to simultaneous approximation and interpolation in more general situations. Indeed, the techniques developed in this paper can be used to obtain analogous results for simultaneous approximation and interpolation by radial-basis functions on a torus, neural networks on a cube, Gaussian networks on Euclidean spaces, etc. We illustrate the main ideas in the context of polynomial interpolation and zonal-function (ZF) networks on the unit sphere; other situations can be treated in the same way.

Precise definitions regarding ZF networks are given in Section 3, but we make some preliminary observations here. A fairly well-developed theory of the approximation capabilities of ZF networks has evolved over the last several years (see [4], 10 for reviews). Some of the appealing properties of the theory include the flexibility to interpolate at scattered points, and the possibility of parallel computation. The convergence of interpolatory ZF networks having a minimal size has been proved only for (target) functions belonging to some "native spaces". Since membership in these native spaces often places a severe restriction on the target function, we wish to explore an "over-sampling" strategy for ZF networks, akin to that in polynomial interpolation. Specifically, we seek networks with size larger than the required minimum for interpolation, but ones which provide both interpolation as well as a (near-optimal) degree of approximation to all continuous functions on the sphere.

\section{MAIN THEOREMS}

For a normed linear space $\mathbb{X}$, we will denote the space of all continuous linear functionals on $\mathbb{X}$ by $\mathbb{X}^{*}$, and the usual norm on this dual space is given by

$$
\left\|x^{*}\right\|_{\mathbb{X}^{*}}:=\sup \left\{\left|x^{*}(x)\right|:\|x\|_{\mathbb{X}} \leq 1\right\} .
$$

Proposition 2.1. Suppose $\mathbb{Y}, \mathbb{X}$ are normed linear spaces, and let $S: \mathbb{Y} \rightarrow \mathbb{X}$ be a continuous linear operator which is bounded below; that is, there exists a positive number $\kappa$ such that

$$
\|y\|_{\mathbb{Y}} \leq \kappa\|S(y)\|_{\mathbb{X}}, \quad \text { for all } y \in \mathbb{Y} .
$$

If $y^{*} \in \mathbb{Y}^{*}$, then there exists $x^{*} \in \mathbb{X}^{*}$ such that

$$
x^{*}(S(y))=y^{*}(y), \quad \text { for every } y \in \mathbb{Y},
$$

and

$$
\left\|x^{*}\right\|_{\mathbb{X}^{*}} \leq \kappa\left\|y^{*}\right\|_{\mathbb{Y}^{*}} .
$$

Proof. Let $\mathbb{X}_{1}$ denote the range of $S$. Since (2.1) ensures that $S$ is injective, $S$ : $\mathbb{Y} \rightarrow \mathbb{X}_{1}$ is invertible; moreover, (2.1) is equivalent to the statement

$$
\left\|S^{-1}\right\| \leq \kappa,
$$


where the norm above is the usual operator norm. Let $y^{*} \in \mathbb{Y}^{*}$, and consider the functional $\left(S^{-1}\right)^{*}\left(y^{*}\right) \in \mathbb{X}_{1}^{*}$, where $\left(S^{-1}\right)^{*}$ is the adjoint of $S^{-1}$. The Hahn-Banach theorem furnishes an $x^{*} \in \mathbb{X}^{*}$ such that

$$
x^{*}\left(x_{1}\right)=\left(S^{-1}\right)^{*}\left(y^{*}\right)\left(x_{1}\right), \quad \text { for every } x_{1} \in \mathbb{X}_{1} ;
$$

equivalently,

$$
x^{*}(S(y))=\left(S^{-1}\right)^{*}\left(y^{*}\right)(S(y))=y^{*}(y), \quad y \in \mathbb{Y},
$$

the last equation resulting from the definition of $\left(S^{-1}\right)^{*}$. Since the Hahn-Banach extension is also norm preserving, we find that

$$
\left\|x^{*}\right\|_{\mathbb{X}^{*}}=\left\|\left(S^{-1}\right)^{*}\left(y^{*}\right)\right\|_{\mathbb{X}_{1}^{*}} \leq\left\|\left(S^{-1}\right)^{*}\right\|\left\|y^{*}\right\|_{\mathbb{Y}^{*}}=\left\|S^{-1}\right\|\left\|y^{*}\right\|_{\mathbb{Y}^{*}} \leq \kappa\left\|y^{*}\right\|_{\mathbb{Y}^{*}},
$$

the final bound obtaining from (2.4).

As a first illustration of the foregoing proposition, we prove a general "quadrature formula". The corollary below plays an important rôle in [7, 6, 8, 9, 11].

Given a finite set of continuous linear functionals on a finite-dimensional normed linear space, we wish to express any continuous linear functional on this space as a linear combination of the functionals in the finite set.

Corollary 2.1. Let $Y$ be a finite-dimensional normed linear space and let $Z:=$ $\left\{y_{1}^{*}, \ldots, y_{N}^{*}\right\} \subset Y^{*}$. Let $T_{Z}: y \in Y \mapsto\left(y_{1}^{*}(y), \ldots, y_{N}^{*}(y)\right) \in \mathbb{R}^{N}$ be an injective operator. Then for every $y^{*} \in Y^{*}$, there exist scalars $w_{1}, \ldots, w_{N}$ such that

$$
y^{*}(y)=\sum_{k=1}^{N} w_{k} y_{k}^{*}(y), \quad y \in Y .
$$

Proof. In Proposition 2.1 we take $Y$ in place of $\mathbb{Y}, \mathbb{R}^{N}$ in place of $\mathbb{X}$, and $T_{Z}$ in place of $S$. The estimate (2.1) follows from the facts that $T_{Z}$ is injective and $Y$ is finite dimensional. Since the dual of $\mathbb{R}^{N}$ may be identified with $\mathbb{R}^{N}$ (with a different norm), we obtain (2.8) from (2.2).

In the context described above, one typically has more functionals in $Z$ than the dimension of $Y^{*}$. In the applications to be discussed in the next section, we wish to interpolate a certain set of functionals by elements of a finite-dimensional space. This may be thought of as dual to the situation in Corollary 2.1 in the sense that the dimension of the space is larger than the number of functionals to be interpolated. More precisely, let $X$ be a normed linear space, with $V \subset X$ a finite-dimensional subspace, and let $Z_{*}$ be the span of $\left\{x_{j}^{*}\right\}_{j=1}^{N} \subset X^{*}$. In general, $N \leq \operatorname{dim}(V)$. We also require that the restriction map $S:\left.z^{*} \in Z_{*} \mapsto z^{*}\right|_{V}$ be injective. If these conditions are satisfied, then one easily sees that for any $f \in X$, we can find a $v \in V$ such that $v$ interpolates $f$ on $Z_{*}$; that is,

$$
z^{*}(v)=z^{*}(f) \quad \text { for every } z^{*} \in Z_{*} .
$$

All this is quite standard. When $N=\operatorname{dim}(V)$, this problem has a unique solution, and there is nothing more to it. However, if we allow the approximation space $V$ to have dimension larger than $N$, we can obtain improved approximation in addition to interpolation. The key theorem to be used in our applications is the following.

Theorem 2.1. Let $X, V, Z_{*}$, and $S$ be as described above. If $\left\|S^{-1}\right\| \leq \kappa$, then for every $f \in X$ and $\kappa_{1}>\kappa$, there exists $\mathrm{T}(f) \in V$ such that

$$
z^{*}(\mathrm{~T}(f))=z^{*}(f) \quad \text { for every } z^{*} \in Z_{*},
$$


and

$$
\|f-\mathrm{T}(f)\|_{X} \leq\left(1+\kappa_{1}\right) \inf _{v \in V}\|f-v\|_{X}
$$

Proof. In Proposition 2.1, we take $\mathbb{Y}=Z_{*}, \mathbb{X}=V^{*}$. The condition on $S^{-1}$ is equivalent to the following:

$$
\left\|z^{*}\right\|_{X^{*}} \leq \kappa\left\|z^{*}\right\|_{V^{*}}, \quad \text { for every } z^{*} \in Z_{*},
$$

which is exactly (2.1). Given any $g \in X$, we may identify it with an element of $\left(Z_{*}\right)^{*}$, via the standard formula $g\left(z^{*}\right)=z^{*}(g), z^{*} \in Z_{*}$. We observe that

$$
\|g\|_{\left(Z_{*}\right)^{*}}=\sup _{\substack{z^{*} \in Z_{*} \\\left\|z^{*}\right\|_{X^{*}} \leq 1}}\left|g\left(z^{*}\right)\right| \leq \sup _{\substack{z^{*} \in X^{*} \\\left\|z^{*}\right\|_{X^{*}} \leq 1}}\left|g\left(z^{*}\right)\right|=\|g\|_{X} .
$$

Now Proposition 2.1 guarantees a $v_{g} \in V=\left(V^{*}\right)^{*}$ such that $g\left(z^{*}\right)=v_{g}\left(S\left(z^{*}\right)\right)$ for every $z^{*} \in Z_{*}$; equivalently, $z^{*}(g)=z^{*}\left(v_{g}\right)$ for every $z^{*} \in Z_{*}$. Moreover, (2.3) and (2.12) lead to the relations

$$
\left\|v_{g}\right\|_{X}=\left\|v_{g}\right\|_{V}=\left\|v_{g}\right\|_{\left(V^{*}\right)^{*}} \leq \kappa\|g\|_{\left(Z_{*}\right)^{*}} \leq \kappa\|g\|_{X} .
$$

Choose $w \in V$ so that $\|f-w\|_{X} \leq\left(\left(1+\kappa_{1}\right) /(1+\kappa)\right) \inf _{v \in V}\|f-v\|_{X}$, and set $\mathbf{T}(f)=w+v_{f-w}$. Then $\mathbf{T}(f)$ satisfies (2.10), and

$$
z^{*}(\mathbf{T}(f))=z^{*}(w)+z^{*}\left(v_{f-w}\right)=z^{*}(w)+z^{*}(f-w)=z^{*}(f)
$$

holds for all $z^{*} \in Z_{*}$.

\section{Applications}

In this section, we apply Theorem 2.1 to obtain results concerning simultaneous approximation and interpolation for a variety of function classes. In what follows, $C(\mathbb{T})$ (respectively, $C[-1,1]$ ) will denote the class of all continuous, $2 \pi$-periodic functions on $\mathbb{R}$ (respectively, all continuous functions from $[-1,1]$ to $\mathbb{R}$ ), equipped with the supremum norm on $[-\pi, \pi]$ (respectively, $[-1,1]$ ).

The following theorem consists of two parts. Part (a) is a periodic version of a theorem of Szabados [15. Theorem 1]. The original result of Szabados in the aperiodic case can be derived from the periodic version, and this is the content of part (b). While the proof in [15] utilizes specialized and intricate properties of interpolating polynomials, etc., our argument stems from abstract and general considerations.

Theorem 3.1. (a) Let $\theta_{1}, \ldots, \theta_{N}$ be distinct points in $[-\pi, \pi], \theta_{N+1}:=\theta_{1}+2 \pi$, $\alpha>0$, and

$$
\min _{1 \leq k \leq N}\left|\theta_{k+1}-\theta_{k}\right|=: \eta .
$$

Then for $f \in C(\mathbb{T})$, there exists a trigonometric polynomial $T$ of degree at most $(1+2 / \alpha)(\pi / \eta)$ such that $f\left(\theta_{j}\right)=T\left(\theta_{j}\right), 1 \leq j \leq N$, and

$$
\|f-T\|_{C(\mathbb{T})} \leq(2+\alpha) \inf \|f-P\|_{C(\mathbb{T})},
$$

where the infimum is taken over all trigonometric polynomials $P$ of degree at most $(1+2 / \alpha)(\pi / \eta)$.

(b) Suppose $\left\{\theta_{k}: 1 \leq k \leq N\right\}$ is a set of points in $[0, \pi]$, and define $x_{k}:=\cos \theta_{k}$. Let $\alpha>0$, and

$$
\min _{1 \leq k \leq N-1}\left|\theta_{k+1}-\theta_{k}\right|=: \eta>0
$$


Then for every $f \in C[-1,1]$, there exists an algebraic polynomial $T$ of degree at most $(1+2 / \alpha)(\pi / \eta)$ such that $f\left(x_{k}\right)=T\left(x_{k}\right), 1 \leq k \leq n$, and

$$
\|f-T\|_{C[-1,1]} \leq(2+\alpha) \inf \|f-P\|_{C[-1,1]},
$$

where the infimum is taken over all algebraic polynomials $P$ of degree at most $(1+2 / \alpha)(\pi / \eta)$.

Proof. (a) In Theorem 2.1 we take $X$ to be the space $C(\mathbb{T})$, and define $z_{j}^{*}(f)=$ $f\left(\theta_{j}\right)$ for $f \in X, j=1, \ldots, N$. Let $Z_{*}$ be the span of $\left\{z_{j}^{*}\right\}_{j=1}^{N}$ and let $M:=$ $\lfloor(1+2 / \alpha)(\pi / \eta)\rfloor$, where $\lfloor t\rfloor$ denotes the largest integer not exceeding the real number $t$. Define $V$ to be the space of all trigonometric polynomials of degree at most $M$. We show that (2.11) holds.

Let $z^{*}=\sum_{j=1}^{N} a_{j} z_{j}^{*} \in Z_{*}$, and let $g \in X$ be the $2 \pi$-periodic, piecewise-linear interpolant to the data $g\left(\theta_{j}\right):=\operatorname{sgn} a_{j}, j=1, \ldots, N$. Then $\|g\|_{X}=1$ and $z^{*}(g)=$ $\sum_{j=1}^{N}\left|a_{j}\right|=\left\|z^{*}\right\|_{X^{*}}$. Further, $|g(\theta)-g(\phi)| \leq(2 / \eta)|\theta-\phi|$ for $\theta, \phi \in[-\pi, \pi]$, so a variant of Jackson's theorem [2] p. 143] supplies a trigonometric polynomial $P \in V$ such that

$$
\|g-P\|_{X} \leq \frac{\pi}{\eta(M+1)}=: \epsilon
$$

Hence, $\|P\|_{X} \leq\|g\|_{X}+\epsilon=1+\epsilon$ and $\left|z^{*}(g)-z^{*}(P)\right| \leq \epsilon\left\|z^{*}\right\|_{X^{*}}$. Since $z^{*}(g)=$ $\left\|z^{*}\right\|_{X^{*}}$, this yields

$$
\left\|z^{*}\right\|_{X^{*}} \leq \frac{1}{1-\epsilon}\left|z^{*}(P)\right| \leq \frac{1+\epsilon}{1-\epsilon}\left\|z^{*}\right\|_{V^{*}}<(1+\alpha)\left\|z^{*}\right\|_{V^{*}}
$$

the final bound coming from the elementary inequality $\lfloor t\rfloor+1>t, t \in \mathbb{R}$. The required result follows from Theorem 2.1

(b) The proof is almost the same as above; we indicate the changes. We take $X$ to be the class of all even functions in $C(\mathbb{T}), Z_{*}$ and $M$ as above. Let $V$ be the class of all even trigonometric polynomials of degree at most $M$. We may assume that $\theta_{1} \leq \ldots \leq \theta_{N}$. Let $g$ be defined as above on $\left[\theta_{1}, \theta_{N}\right]$. Extend $g$ to $[-\pi, \pi]$ via the equations $g(\theta) \equiv g\left(\theta_{1}\right), \theta \in\left[0, \theta_{1}\right], g(\theta) \equiv g\left(\theta_{N}\right), \theta \in\left[\theta_{N}, \pi\right]$, and $g(\theta)=g(-\theta)$, $\theta \in[-\pi, 0]$. Then $g$ satisfies all the conditions in part (a), whose proof then provides a trigonometric polynomial $Q$ with $\|g-Q\|_{C(\mathbb{T})} \leq \epsilon$. Since $g$ is even, the polynomial $P(\theta)=(Q(\theta)+Q(-\theta)) / 2 \in V$ satisfies $\|g-P\|_{X} \leq \epsilon$. Proceeding as in part (a), we obtain for every $f \in X$ an even trigonometric polynomial $T^{*}$ that interpolates $f$ at $\theta_{k}, 1 \leq k \leq N$, and has the desired approximation property. In view of the standard correspondence between $C[-1,1]$ and $X$, and $V$ and the class of all algebraic polynomials of degree $M$, the proof is complete.

Instances where the Jackson property holds abound in approximation theory. Therefore, we find it worthwhile to extend the proof of the preceding theorem to cover more general situations.

Suppose $d \geq 1$ is an integer, $K \subset \mathbb{R}^{d}$ is a closed set, and $C(K)$ is the space of all real-valued, continuous, and bounded functions on $K$, equipped with the supremum norm. If $\mu$ is a (nonnegative) Borel measure on $K$, and $x \in K$, we say that $x$ is a mass point of $\mu$ if $\mu(U)>0$ for every neighborhood $U$ of $x$. The support of $\mu$, denoted by $\operatorname{supp}(\mu)$, is the closure of the set of all mass points of $\mu$. Let $r \geq 1$ be an integer, and let $\mathcal{D}$ be a linear (partial) differential operator of order $r$ defined for a subset of $C(K)$, and having coefficient functions in $C(K)$. We say that a sequence 
$\left\{V_{n}\right\}$ of subspaces of $C(K)$ has the Jackson property with respect to $\mathcal{D}$ if for every $f$ in the domain of $\mathcal{D}$, and $n \geq 1$, one has

$$
\inf _{v \in V_{n}}\|f-v\|_{C(K)} \leq A n^{-r}\left\{\|f\|_{C(K)}+\|\mathcal{D}(f)\|_{C(K)}\right\},
$$

where $a$ is a positive constant, independent of $n$ and $f$. We emphasize that the dimension of $V_{n}$ is not necessarily $n$. We shall find it convenient to let the symbol $V_{x}$ denote the space $V_{\lceil x\rceil}$, where $\lceil x\rceil$ denotes the smallest integer not less than $x$.

Theorem 3.2. Suppose that $\mu_{1}, \ldots, \mu_{N}$ are compactly supported Borel measures on $K \subseteq \mathbb{R}^{d}$, and that $S_{j}:=\operatorname{supp}\left(\mu_{j}\right), j=1, \ldots, N$, are mutually disjoint. Assume that

$$
\frac{\beta}{n} \leq \eta:=\min _{1 \leq i, j \leq N} \operatorname{dist}\left(S_{i}, S_{j}\right) \leq \Delta,
$$

for some positive integer $n$ and positive constants $\beta$ and $\Delta$ (which may depend on $K$ and $d$, but not on $N, \mu_{j}$ 's or $S_{j}$ 's). Let $r \geq 1$ be an integer, and let $\mathcal{D}$ be a linear (partial) differential operator of order $r$ defined for a subset of $C(K)$, and having coefficient functions in $C(K)$. Further, let $\left\{V_{k}\right\}$ be a sequence of finite-dimensional subspaces of $C(K)$ having the Jackson property with respect to $\mathcal{D}$. If $\alpha>0$, there exists a positive constant $C:=C(\alpha, \beta, d, \Delta, \mathcal{D})$ with the following property: For every $f \in C(K)$, there exists $\mathrm{T}_{n}(f) \in V_{C n}$ such that

$$
\int f d \mu_{j}=\int \mathrm{T}_{n}(f) d \mu_{j}, \quad j=1, \ldots, N
$$

and

$$
\left\|f-\mathrm{T}_{n}(f)\right\|_{C(K)} \leq(2+\alpha) \inf _{v \in V_{C n}}\|f-v\|_{C(K)} .
$$

Proof. We assume, without loss of generality, that the distance in (3.14) pertains to the $\ell^{\infty}$ metric in $\mathbb{R}^{d}$. Consider the functionals $x_{j}^{*}$ on $C(K)$ defined by $x_{j}^{*}(f)=$ $\int f d \mu_{j}$. In Theorem 2.1, we take $X=C(K)$, and $Z_{*}$ as the space spanned by the functionals $x_{j}^{*}$. It remains to find a space $V$ for which (2.11) holds (for a suitable $\kappa)$. Let $U(\mathbf{x}, \delta)$ denote an open cube which is centred at $\mathbf{x}$ and whose sides are of length $\delta$. We decompose the space $\mathbb{R}^{d}$ into a grid consisting of cubes with sides of length $\eta / 4$. Suppose $1 \leq j \leq N$, and let $\left\{U\left(\mathbf{x}_{k}^{j}, \eta / 4\right)\right\}_{k=1}^{m(j)}$ be the finite set of cubes from this grid which covers $S_{j}$; define $B_{k}^{j}:=U\left(\mathbf{x}_{k}^{j}, \eta / 2\right), 1 \leq k \leq m(j)$. Let $\phi$ be an infinitely differentiable function on $\mathbb{R}^{d}$, such that $0 \leq \phi(\mathbf{x}) \leq 1$ for $\mathbf{x} \in \mathbb{R}^{d}$, $\phi(\mathbf{x})=1$ for $\mathbf{x} \in U(\mathbf{0}, 1 / 2)$, and $\phi(\mathbf{x})=0$ if $\mathbf{x} \notin U(\mathbf{0}, 1)$. For $1 \leq k \leq m(j)$, write $\phi_{k j}(\mathbf{x})=\phi\left(2\left(\mathbf{x}-\mathbf{x}_{k}^{j}\right) / \eta\right)$, and, following [14, Theorem 6.20], define

$$
f_{j}:=1-\prod_{k=1}^{m(j)}\left(1-\phi_{k j}\right), \quad 1 \leq j \leq N .
$$

Then each $f_{j}$ is infinitely differentiable, $f_{j}(\mathbf{x})=1$ if $\mathbf{x} \in S_{j}, f_{j}(\mathbf{x})=0$ if $\mathbf{x} \notin$ $\bigcup_{k=1}^{m(j)} B_{k}^{j}$, and $\left\|f_{j}\right\|_{C(K)}=1$. Since the number of $B_{k}^{j}$, s intersecting any given $B_{l}^{j}$ can be bounded by a number which depends only on $d$, we find that $\left\|\mathcal{D} f_{j}\right\|_{C(K)} \leq$ $B_{1} \eta^{-r}$ for some constant $B_{1}=B_{1}(d)>0$. Hence, (3.14) (specifically, the inequality $\eta^{r} \leq \Delta^{r}$ ) leads to the bound $\left\|f_{j}\right\|_{C(K)}+\left\|\mathcal{D} f_{j}\right\|_{C(K)} \leq B \eta^{-r}$ for some constant $B=B(d, \Delta, r)>0$.

Now let $z^{*}=\sum_{j=1}^{N} a_{j} x_{j}^{*} \in Z_{*}$, and define $g=\sum_{j=1}^{N}\left(\operatorname{sgn} a_{j}\right) f_{j}$. Since the sets $S_{j}$ are separated by a distance of $\eta$ (see (3.14) $), B_{k}^{j} \cap B_{l}^{i}=\emptyset$ whenever $i \neq j, 1 \leq$ 
$k \leq m(j)$, and $1 \leq l \leq m(i)$. Therefore, the $f_{j}$ 's are disjointly supported. It follows that $\|g\|_{C(K)}=1$ and $\|g\|_{C(K)}+\|\mathcal{D} g\|_{C(K)} \leq B \eta^{-r}$. Furthermore, $\left\|z^{*}\right\|_{C(K)^{*}}=$ $z^{*}(g)=\sum_{j=1}^{N}\left|a_{j}\right| \mu_{j}\left(S_{j}\right)$. Since the sequence $\left\{V_{k}\right\}$ satisfies the Jackson property with respect to $\mathcal{D}$, we can find for every $C>0, P_{C n} \in V_{C n}$ with the property that

$$
\left\|g-P_{C n}\right\|_{C(K)} \leq A(C n)^{-r}\left\{\|g\|_{C(K)}+\|\mathcal{D} g\|_{C(K)}\right\} \leq \frac{A B}{(C \eta n)^{r}} \leq \frac{A B}{(C \beta)^{r}} .
$$

Let $C:=(1 / \beta)\left[A B\left(\frac{5+\alpha}{\alpha}\right)\right]^{1 / r}$, so that

$$
\left\|g-P_{C n}\right\| \leq \frac{\alpha}{5+\alpha} .
$$

We now repeat the argument from the last part of the proof of Theorem 3.1(a), and arrive at the key estimate

$$
\left\|z^{*}\right\|_{C(K)^{*}} \leq\left(1+\frac{2 \alpha}{5}\right)\left\|z^{*}\right\|_{V_{C n}^{*}}
$$

Choosing $V=V_{C n}$, we find that all the conditions of Theorem 2.1 are satisfied.

We point out some implications of Theorem 3.2 in two examples.

Our first example concerns approximation on a sphere by polynomials. Let $q \geq 1$ be an integer, $\mathbb{S}^{q}$ be the (surface of the) unit sphere in $\mathbb{R}^{q+1}$. Let $\Pi_{n}^{q}$ be the class of all polynomials in $q+1$ variables having total degree $n$ or less, restricted to $\mathbb{S}^{q}$; these are the spherical harmonics of degree at most $n$. Let $\mathcal{C}$ be a finite set of distinct points on $\mathbb{S}^{q}$. The mesh norm $\delta_{\mathcal{C}}$ and the minimal separation $\eta_{\mathcal{C}}$ are defined by equations

$$
\delta_{\mathcal{C}}:=\sup _{\mathbf{x} \in \mathbb{S}^{q}} \operatorname{dist}(\mathbf{x}, \mathcal{C}), \quad \eta_{\mathcal{C}}:=\min _{\mathbf{x}, \mathbf{y} \in \mathcal{C}} \operatorname{dist}(\mathbf{x}, \mathbf{y}),
$$

where we take the distance to be the geodesic distance between points on the sphere.

Corollary 3.1. Let $\mathcal{C}=\left\{\xi_{1}, \ldots, \xi_{N}\right\}$ be a finite set of distinct points on $\mathbb{S}^{q}$. Assume that $\eta_{\mathcal{C}} \geq \beta / n$ for some $\beta>0$ and integer $n$. Given $\alpha>0$, there exists a constant $C:=C(\alpha, \beta, q)$ with the following property: for every $f \in C\left(\mathbb{S}^{q}\right)$, there exists $P \in \Pi_{C n}^{q}$ such that

$$
P\left(\xi_{j}\right)=f\left(\xi_{j}\right), \quad 1 \leq j \leq N,
$$

and

$$
\|f-P\|_{C\left(\mathbb{S}^{q}\right)} \leq(2+\alpha) \inf _{R \in \Pi_{C n}^{q}}\|f-R\|_{C\left(\mathbb{S}^{q}\right)} .
$$

Proof. We apply Theorem 3.2 with $K=\mathbb{S}^{q}$ and $\mu_{j}$ the Dirac measure of mass one concentrated at $\xi_{j}, 1 \leq j \leq N$. The Laplace-Beltrami operator is a partial differential operator of order 2 with coefficients continuous on $\mathbb{S}^{q}$, and Pawelke [13. has proved that the sequence of spaces $\left\{\Pi_{n}^{q}\right\}$ satisfies the Jackson property with respect to this operator.

Our final application is to the theory of approximation of functions on the sphere by zonal-function networks. Let $\phi:[-1,1] \rightarrow \mathbb{R}$ be a continuous function. A zonalfunction $(Z F)$ network is a function of the form $\mathbf{x} \mapsto \sum_{k=1}^{n} a_{k} \phi\left(\mathbf{x} \cdot \xi_{k}\right)$, where $\xi_{k} \in \mathbb{S}^{q}$. There are several results concerning interpolation by ZF networks, which is guaranteed for positive definite functions $\phi$, and the interpolation processes are guaranteed to converge, as long as the target function belongs to a suitable class, 
to wit, the so-called native space. But the latter is often quite small, so one desires processes which provide both interpolation and approximation in larger (e.g. Sobolev) classes. Corollary 3.2 below shows that it is possible to meet these requirements for arbitrary scattered data on the sphere, even with very mild conditions on the function $\phi$, provided the size of the network is allowed to be sufficiently larger than the number of interpolatory constraints.

Before stating our result, we recall some notation and known results. For further motivation and details, we refer to 9 . By $\mathcal{P}_{\ell}(q+1 ; \cdot)$, we denote the orthogonal polynomial of degree $\ell$ satisfying the orthogonality relations

$$
\int_{-1}^{1} \mathcal{P}_{\ell}(q+1 ; x) \mathcal{P}_{k}(q+1 ; x)\left(1-x^{2}\right)^{\frac{q}{2}-1} d x=\sqrt{\pi} \frac{\Gamma(q / 2)}{\Gamma((q+1) / 2) d_{\ell}^{q}} \delta_{\ell, k},
$$

where

$$
d_{\ell}^{q}:=\left\{\begin{array}{cl}
\frac{2 \ell+q-1}{\ell+q-1}\left(\begin{array}{c}
\ell+q-1 \\
\ell
\end{array}\right), & \text { if } \ell \geq 1 \\
1, & \text { if } \ell=0
\end{array}\right.
$$

In the sequel, the positive integer $q$ will be held fixed. For a continuous function $\phi:[-1,1] \rightarrow \mathbb{R}$, and $\ell=0,1,2, \ldots$, we write

$$
b_{\ell}(\phi):=\frac{\Gamma((q+1) / 2) d_{\ell}^{q}}{\sqrt{\pi} \Gamma(q / 2)} \int_{-1}^{1} \mathcal{P}_{\ell}(q+1 ; x) \phi(x)\left(1-x^{2}\right)^{\frac{q}{2}-1} d x .
$$

We assume that $b_{\ell}(\phi) \neq 0$ for any $\ell=0,1,2, \ldots$, and define

$$
m_{N}:=\min _{0 \leq \ell \leq N} \frac{\left|b_{\ell}(\phi)\right|}{d_{\ell}^{q}}, \quad N=1,2, \ldots
$$

The degree of approximation of $\phi$ by polynomials of degree at most $M$ is given by

$$
E_{M}(\phi):=\min \|\phi-P\|_{C[-1,1]},
$$

where the minimum is taken over all polynomials $P$ of degree at most $M$. Let $k_{q}$ be the least integer exceeding $(q-1) / 2$. We define a sequence $\left\{M_{n}=M_{n}(\phi)\right\}$ by

$$
E_{M_{n}}(\phi) \leq \frac{m_{\left(k_{q}+1\right) n-1}}{n^{2}}
$$

Finally, given a set $\mathcal{C}$ of distinct points on $\mathbb{S}^{q}$, the space $V_{\phi, \mathcal{C}}$ of $\mathrm{ZF}$ networks is defined by

$$
V_{\phi, \mathcal{C}}:=\operatorname{span}\{\phi((\cdot) \cdot \xi): \xi \in \mathcal{C}\}
$$

We are now in a position to state our result regarding simultaneous approximation and interpolation by ZF networks.

Corollary 3.2. Let $\phi:[-1,1] \rightarrow \mathbb{R}$ be a continuous function such that $b_{\ell}(\phi) \neq 0$ for every $\ell=0,1,2, \ldots$ Suppose $\mathcal{C}_{1}^{*}$ is a set of distinct points on $\mathbb{S}^{q}$, and let $n \geq 1$ be an integer such that $\eta_{\mathcal{C}_{1}^{*}} \geq \beta / n$ for some $\beta>0$. Then there exist numbers $L, \alpha>0$ such that for any set $\mathcal{C}$ of distinct points on $\mathbb{S}^{q}$ with

$$
L\left(k_{q}+2\right) n+M_{L n}(\phi) \leq \alpha \delta_{\mathcal{C}}^{-1}
$$


and every $f \in C\left(\mathbb{S}^{q}\right)$, there exists a $Z F$ network $\mathcal{N} \in V_{\phi, \mathcal{C}}$ such that

$$
\mathcal{N}(\xi)=f(\xi), \quad \xi \in \mathcal{C}_{1}^{*}
$$

and

$$
\|f-\mathcal{N}\|_{C(\mathbb{S} q)} \leq c \inf _{P \in V_{\phi, \mathcal{C}}}\|f-P\|_{C\left(\mathbb{S}^{q}\right)},
$$

for some constant $c$.

Proof. In Theorem [3.2, we take $K$ to be $\mathbb{S}^{q}$, and for each $\xi \in \mathcal{C}_{1}^{*}$, we let $\mu_{\xi}$ be the measure of mass one concentrated at $\xi$. The following was established in [9]: There exist $N, \alpha>0$ such that if $\left\{\mathcal{C}_{n}\right\}_{n \geq N}$ is a sequence of sets of distinct points on $\mathbb{S}^{q}$ with $\left(k_{q}+2\right) n+M_{n}(\phi) \leq \alpha \delta_{\mathcal{C}_{n}}^{-1}$, then the sequence of spaces $\left\{V_{\phi, \mathcal{C}_{n}}\right\}_{n \geq N}$ satisfies the Jackson property with respect to the Laplace-Beltrami operator (cf. [9, Formulae $(3.2),(4.13)])$.

Although Corollary 3.2 does not specify the construction of the network $\mathcal{N}$, it is reasonable to expect that the network can be constructed using adaptations of the least square technique, subject to the constraints (3.25).

\section{REFERENCES}

[1] S. Bernstein, Sur une modification de la formule d'interpolation de Lagrange, Comm. Soc. Math. Kharkov, 5 (1932), 49-57.

[2] E. W. Cheney, "Introduction to approximation theory", McGraw-Hill Inc., New York, 1966. MR 36:5568

[3] P. Erdős, On some convergence properties of the interpolation polynomials, Ann. Math., 44 (1943), 330-337. MR 4:273e

[4] G. E. Fasshauer and L. L. Schumaker, Scattered data fitting on the sphere, in "Mathematical Methods for Curves and Surfaces II", M. Dæhlen, T. Lyche, and L. L. Schumaker (eds.), Vanderbilt University Press, Nashville \& London, 1998, 117-166. MR 99d:41054

[5] G. Grünwald, On the theory of interpolation, Acta Math., 75 (1943), 219-245. MR 7:157e

[6] K. Jetter, J. Stöckler, and J. D. Ward, Error estimates for scattered data interpolation, Math. Comp., 68 (1999), 733-747. MR 99i:41032

[7] K. Jetter, J. Stöckler, and J. D. Ward, Norming sets and spherical cubature formulas, in "Computational Mathematics", Z. Chen, Y. Li, C. Micchelli, and Y. Xu (eds.), Marcel Dekker, New York, 1998, 237-245. MR 99i:65022

[8] H. N. Mhaskar, F. J. Narcowich, and J. D. Ward, Spherical Marcinkiewicz-Zygmund inequalities and positive quadrature, Math. Comp., 70 (2001), 1113-1130. CMP 2001:11

[9] H. N. Mhaskar, F. J. Narcowich, and J. D. Ward, Approximation properties of zonal function networks using scattered data on the sphere, Adv. Comput. Math., 11 (1999), 121-137. CMP 2000:06

[10] H. N. Mhaskar, F. J. Narcowich, and J. D. Ward, Representing and analyzing scattered data on spheres, in "Multivariate Approximation and Applications", N. Dyn, D. Leviaton, D. Levin, and A. Pinkus (eds.), Cambridge Univ. Press, Cambridge, U. K., 2001.

[11] H. N. Mhaskar and J. Prestin, On the detection of singularities of a periodic function, Adv. Comput. Math., 12 (2000), 95-131. MR 2001a:42003

[12] I. P. Natanson, "Constructive function theory, Vol. 3", Frederick Ungar Publishing Co., New York, 1965. MR 33:4529c

[13] S. Pawelke, Über die Approximationsordnung bei Kugelfunktionen und algebraischen Polynomen, Tôhoku Math. J., 24 (1972), 473-486. MR 48:774

[14] W. Rudin, "Functional analysis", McGraw-Hill Inc., New York, 1973. MR 51:1315

[15] J. Szabados, On some convergent interpolatory polynomials, in "Fourier Analysis and Approximation Theory", Colloq. Math. Soc. János Bolyai, Vol. 19, North-Holland Publishing Co., Amsterdam, 1976, 805-815. MR 81g:41009 
[16] J. Szabados and P. Vertesi, "Interpolation of functions", World Scientific Publishing Co., Singapore, 1990. MR 92j:41009

[17] P. Vertesi, Convergent interpolatory processes for arbitrary systems of nodes, Acta Math. Acad. Sci. Hungar., 33 (1979), 223-234. MR 80g:41007

Department of Mathematics, California State University, Los Angeles, California 90032

Center for Approximation Theory, Department of Mathematics, Texas A\&M UniverSity, College Station, Texas 77843-3368

E-mail address: fnarc@math.tamu.edu

Center for Approximation Theory, Department of Mathematics, Texas A\&M UniverSity, College Station, Texas 77843-3368

E-mail address: n.sivakumar@math.tamu.edu

Center for Approximation Theory, Department of Mathematics, Texas A\&M University, College Station, Texas 77843-3368

E-mail address: jward@math.tamu.edu 